\title{
Stratégies de traduction des phrases (dé)figées dans La Scomparsa, version italienne de $L a$ Disparition de Georges Perec
}

\author{
Gerardo ACERENZA \\ Università degli Studi di Trento \\ Italie
}

\begin{abstract}
Résumé :
Dans cette étude nous nous proposons d'analyser les stratégies que le traducteur Piero Falchetta a mises en œuvre pour rendre en italien les nombreuses phrases (dé)figées par Georges Perec dans La Disparition. Pour répondre à la contrainte lipogrammatique choisie pour l'écriture de La Disparition, Georges Perec a réinventé un grand nombre d'expressions figées et de proverbes. Par exemple, l'expression « être/se mettre sur son trente-et-un » a été transformée par Perec en «mis sur son vingt-huit plus trois» $(1989,130)$. Pour respecter la contrainte lipogrammatique (absence de la voyelle « $E »$ ), Perec propose une réécriture de l'expression qui permet tout de même aux lecteurs de la reconnaître en tant que telle et d'en apprécier l'originalité. Comment le traducteur Piero Falchetta a-t-il rendu en italien ces expressions réinventées ? A-t-il mis en place des stratégies particulières pour traduire les séquences détournées par Georges Perec dans La Disparition?
\end{abstract}

Mots-clés : traduction, contrainte, Perec, La Disparition, La Scomparsa.

\begin{abstract}
:
The aim of this study is to understand the strategies applied by the translator Piero Falchetta to translate into Italian the many expressions (dé)figées by Georges Perec into La Disparition. To satisfy the lipogram constraint on " $E$ " chosen for the writing of La Disparition, Georges Perec has reinvented a large number of fixed expressions. For exemple, the french expression "être/se mettre sur son trente-et-un" was transformed by Perec into "mis sur son vingt-huit plus trois" (1989, 130). To respect the lipogram constraint, Perec proposes a rewriting of the expression which still allows French readers to recognize it and appreciate its originality. How did the translator Piero Falchetta translate these reinvented expressions into Italian? Has he put in place specific strategies to translate the sequences reinvented by Georges Perec into La Disparition?
\end{abstract}

Keywords : translation, constraint, Perec, La Disparition, La Scomparsa.

\section{Introduction}

La Disparition, la première œuvre oulipienne de Perec, est souvent citée lorsqu'on évoque la littérature à contrainte et l'Ouvroir de Littérature Potentielle. Cependant, il s'agit d'un roman qu'un 
grand nombre de lecteurs n'a pas vraiment lu du début à la fin. En parlant de ce texte, on s'étonne d'habitude de l'aspect lipogrammatique du roman, de l'incroyable capacité de l'auteur à écrire 316 pages sans jamais utiliser la voyelle « $E$ », la lettre la plus employée de la langue française. Toutefois, on est souvent incapable de citer le nombre de chapitres qui composent le roman ou le nom du personnage qui apparaît au premier chapitre et, plus encore, on passe à côté d'un grand nombre d'acrobaties linguistiques mises en œuvre par Perec pour respecter la contrainte lipogrammatique, comme les réinventions des phrases figées. Dans Palimpsestes, Gérard Genette $(1982,60)$ avait remarqué que «ce roman étrange et contraint » de Perec « invite [...] simplement à y percevoir l'absence de $E »$.

Le lipogramme est présent dans la structure même du roman qui est divisé en 26 chapitres regroupés en 6 parties, précédés d'un " avant-propos » et suivis d'un «post-scriptum ». Chaque partie représente une voyelle de l'alphabet latin, la sixième comprenant l'«y voyelles de l'alphabet français et la deuxième partie n'existe pas non plus, car la lettre « $E »$ est la deuxième voyelle de l'alphabet. Cependant, si la lettre « $E »$ ne figure pas dans le texte, grâce à son caractère métatextuel, le roman présente un grand nombre de passages et de jeux de mots qui évoquent cette voyelle, comme ces images que nous citons à titre d'exemple : « Ainsi, parfois, un rond, pas tout à fait clos, finissant par un trait horizontal : on aurait dit un grand G vu dans un miroir » (Perec [1969] 1989, 19). Ou encore cette autre image qu'on retrouve au début du texte dans la lettre d'Anton Voyl: "trois traits horizontaux (dont l'un au moins paraissait plus court) qu'un gribouillis confus barrait » (Perec [1969] 1989, 55). Le roman de Perec respecte ainsi la règle formulée par Roubaud $(1988,90)$ : " Un texte écrit suivant une contrainte parle de cette contrainte ».

Un grand nombre de noms propres illustrent également la contrainte lipogrammatique que l'auteur s'est imposée. Le nom du premier personnage qui apparaît dans le roman, Anton Voyl, fait entendre la lettre « $E$ » que l'écrivain n'a pas utilisée : "voyelle atone », voyelle qu'on n'entend pas, avec une légère modification du prénom "Anton/Atone ». Même le nom du personnage "Amaury Conson » évoque l'absence de la lettre « $E$ »: consonne où il manque le «ne » de la fin avec la voyelle « $E$ ». En outre, les lettres initiales des noms des personnages Augustus B. Clifford et de son fils adoptif Douglas Haig (Perec [1969] 1989, 102) renvoient à l'ordre des lettres de l'alphabet français : ABCD, mais la cinquième lettre est rendue par la diphtongue « $\mathbf{a i} »$ et non pas par la voyelle « $E »$. 
Par ces prémisses, on comprend avec netteté que la traduction de ce texte n'est pas une tâche aisée, car le lipogramme ne concerne pas seulement l'absence de la lettre « $E$ », mais oriente et structure tout le roman.

\section{Sur les traductions de La Disparition}

Il existe plusieurs traductions de La Disparition (14 achevées). Presque toutes ont proposé une version qui respecte la contrainte lipogrammatique que Perec s'est imposée (absence de la voyelle « $E »)$, comme la version anglaise $A$ Void (1994), jeu de mots sur « un vide » et « éviter » de Gilbert Adair ${ }^{1}$, les versions inédites toujours anglaises Vanish'd! (1989) de John Lee, A vanishing de Ian Monk et Omissions de Julian West qui ne sont pas datées, la traduction allemande Anton Voyls Fortgang (1986) d'Eugen Helmlé, La Scomparsa (1995) de Piero Falchetta en italien, Ispario (2012) de Vanda Mikšić et Morena Livakovic en croate, Disparitia (2010) en roumain par Şerban Foarţă qui préserve la contrainte de l'original, Försvinna (2000) en suédois par Sture Pyk, Kayboluş (2006) en turc par Cemal Yardimci, 't Manco (2009) en néerlandais par Guido Van de Wieil, alors que les traductions Ischezanie (2005) en russe de Valéry Kislov et El Secuestro (1997) en espagnol par Marisol Arbués, Mercé Burrel, Marc Parayre, Hermes Salceda et Regina Vegas ont changé la nature du lipogramme. La version espagnole propose le lipogramme en « $A$ », car en espagnol la voyelle « $A$ » est la lettre la plus employée, tandis que la traduction russe propose le lipogramme en « $O$ » qui est la voyelle la plus utilisée dans cette langue. Il existe encore d'autres traductions, comme Enmetsu (2010) en japonais de Shuichiro Shiotsuka qui propose le lipogramme en « $I$ » et une version en ukrainien qui est sans lipogramme.

Une fois réglé le choix de la contrainte lipogrammatique, le « $A$ » pour la version espagnole, le « $O$ » pour le russe, le « $I »$ pour la version japonaise, ces trois traducteurs ont également dû réorganiser le nombre des chapitres et le nombre des parties qui regroupent les chapitres. En effet, le nombre des chapitres correspond au nombre des lettres de l'alphabet latin et il est étroitement lié à la disparition de la voyelle $E »$. Perec, répétons-le encore une fois, a éliminé le cinquième chapitre qui correspondait pour lui à la cinquième lettre de l'alphabet (le « $E$ ») et la deuxième partie qui correspondait à la deuxième voyelle de

${ }^{1}$ Selon Sara R. Greaves (2000, 115), dans sa traduction on retrouve le rythme du « roman policier, ainsi que, dans le style, une certaine verve. Son titre calembouresque est une bonne trouvaille. Mais La Disparition est beaucoup plus que la parodie d'un roman policier; le contexte anglo-américain dans lequel il replace le roman est construit de façon plutôt arbitraire ». 
l'alphabet (le «E»). À cause de cette contrainte métatextuelle, le traducteur japonais propose par exemple 39 chapitres, nombre qui correspond dans les alphabets syllabiques " hiragana et katakana » aux nombres de syllabes qui ne présentent pas le phonème « $I$ » sur un total de 48. Les mêmes changements ont été nécessaires pour la traduction russe de Valéry Kislov. L'alphabet russe étant formé de 33 lettres, la version russe propose 33 chapitres regroupés en 10 parties, car il existe 10 voyelles. Les traducteurs espagnols quant à eux, à cause du lipogramme en " $A$ », ont donc éliminé le premier chapitre et également la première partie, puisque le « $A$ » est la première lettre de l'alphabet et également la première voyelle. Il s'agit juste de petits exemples qui montrent comment la contrainte lipogrammatique et métatextuelle a entraîné toute une série de transformations selon la nature et la potentialité de la langue d'arrivée.

Presque tous les traducteurs de La Disparition de Perec ont expliqué lors de colloques, tables rondes ou par le moyen de préfaces, postfaces ou articles publiés dans des revues, les stratégies adoptées pour la traduction de cette œuvre qui a souvent été considérée comme illisible et donc intraduisible. Le caractère illisible du roman encourage souvent les éditeurs à proposer un résumé de chaque chapitre à la fin du roman, comme la version italienne $L a$ Scomparsa. À propos de l'intraduisibilité, l'écrivain et traducteur oulipien étatsunien Harry Mathews écrivait à ce propos dans un compte rendu un peu daté des années quatre-vingts :

«La Disparition doit rester sans traduction, non à cause de quelque obstacle technique, mais parce qu'une unité indissoluble détermine chaque aspect du livre (jusqu'à sa ponctuation, parfois). On ne saurait que recréer le roman dans une langue étrangère en inventant de nouveaux personnages, de nouveaux événements, une nouvelle texture, bref en écrivant un tout autre livre. En ceci, aussi, La Disparition peut servir de provocation et d'exemple. » $(1980,31)$

Toutefois, Harry Mathews a été démenti, car on compte jusqu'aujourd'hui déjà quatorze traductions dans plusieurs langues.

\section{Réception de l'œuvre de Perec en Italie}

Dans les années soixante en Italie, il n'existait aucun écrivain qui écrivit en s'imposant une contrainte lipogrammatique ou une contrainte tout court. L'Oplepo, l'Opificio di Letteratura Potenziale, voit le jour seulement en 1990, trente ans après la création de l'Oulipo en France par François Le Lionnais et Raymond Queneau. C'est sur l'île de Capri, dans le golfe de Naples, que le 3 novembre 
1990 deux professeurs universitaires de littérature française, Domenico D'Oria et Ruggero Campagnoli, avec la collaboration de Raffaele Aragona, un ingénieur-journaliste, fondent l'Oplepo, le petit frère italien de l'Oulipo. Jusque-là, il n'existait pas en Italie une vraie tradition de littérature à contrainte. Il y avait la revue de littérature satirique Il Caffé, fondée en 1953 par Giambattista Vicàri, dans les pages de laquelle des auteurs italiens comme Calvino, Pasolini, Zanzotto, Pavese, Gadda et Buzzati publiaient des textes sur des auteurs français et proposaient des extraits de traductions de Céline, Queneau, Roussel, Perec et Michaux. C'est grâce aux pages de cette revue éclectique et expérimentale qu'on a commencé à lire les oulipiens et à connaître la littérature à contrainte.

Perec a beaucoup été traduit en Italie : 16 des 21 ouvrages publiés avant la mort de l'écrivain ont été traduits par différentes maisons d'éditions italiennes, ce qui correspond à $76 \%$ de son œuvre (Bloomfield, 41). Les Choses, par exemple, a connu trois éditions différentes : d'abord chez Mondadori (1966), puis chez Rizzoli (1986) et en 2011 chez Einaudi. Toutefois, Les Revenentes, le roman écrit avec la contrainte du monovocalisme en « $E$ » n'a pas encore été traduit et l'on peut imaginer les raisons.

La Disparation a été entièrement traduit en italien par Piero Falchetta. Il existe aussi de longs passages inédits du roman traduits par l'écrivain Gianni Celati et par le traducteur Bruno Chiaranti. La Scomparsa, la version de Piero Falchetta parue en 1995, que nous analysons ici pour illustrer la stratégie utilisée pour traduire les phrases figées réinventées par Perec, est donc la seule édition publiée. Le traducteur italien est ainsi devenu membre in aeternum de l'Opleplo. Cependant, Piero Falchetta n'est pas un professionnel de la traduction : il travaille comme historien de la navigation et de la cartographie dans une bibliothèque de Venise. Lorsqu'il est interviewé sur la traduction de La Disparition, il répète toujours qu'il n'a été, dans toute sa vie, qu'une seule fois vrai traducteur. Pour cette traduction de Perec, il s'est vu décerner le prestigieux Prix Monselice en 1996. Il est également essayiste et spécialiste de poésie italienne et notamment du poète Andrea Zanzotto.

Comme nous l'avons déjà souligné, la version de Piero Falchetta respecte la contrainte lipogrammatique que Perec s'est imposée, c'est-à-dire l'absence de la voyelle « $E$ ». Le traducteur italien a également gardé l'organisation des chapitres et des parties du texte de départ. À la lecture de La Disparition, notre attention est tombée, entre autres choses, sur les réinventions que l'écrivain a opérées sur un grand nombre de phrases figées de la langue française. Nous pouvons avancer l'hypothèse que Perec a délibérément choisi un grand nombre de structures comprenant la lettre « $E$ » pour ensuite prendre plaisir à les détourner à sa guise. 
D'où l'intérêt de voir la stratégie adoptée par Piero Falchetta pour rendre ces phrases en italien.

Par exemple, dans le passage suivant, le lecteur français reconnaît malgré la modification une phrase idiomatique de la langue française très connue : "Chacun gagna son local privatif, puis réapparut, un instant plus tard, mis sur son vingt-huit plus trois » (Perec [1969] 1989, 130). Pour respecter la contrainte imposée, Perec a modifié l'expression figée " se mettre sur son trente-et-un » qui contient des « $E$ ». La question que nous nous sommes posée est la suivante: Quelle stratégie Piero Falchetta a-t-il utilisée pour rendre ces phrases figées réinventées ? A-t-il procédé, sur la langue d'arrivée, au même travail que Perec avait fait sur la langue française? Il est légitime de se poser ces questions, étant donné que les acrobaties linguistiques mises en ouvre par Perec «procure[nt] au lecteur un amusement devant la capacité de maniement de la langue » (Longhi 2013, 238).

\section{Les phrases figées réinventées par Perec}

Les phrases figées sont généralement appelées expressions idiomatiques, locutions, expressions figurées, idiomes, phrases toutes faites, clichés, manières de dire. Pour le Petit Robert (1996), une expression ou locution figée est une phrase "dont on ne peut changer aucun des termes, et dont le sens global ne correspond pas au sens des différents composants ». Selon le Dictionnaire de linguistique de Dubois (1994, 214), le figement est un «processus linguistique qui, d'un syntagme dont les éléments sont libres, fait un syntagme dont les éléments ne peuvent être dissociés ». Quant à Alain Rey et Sophie Chantreau (1989, V), auteurs du Dictionnaire des expressions et locutions, ils remarquent qu'en plus des mots, il existe dans une langue "des groupes de mots plus ou moins imprévisibles, dans leur forme parfois, et toujours dans leur valeur ». Ces définitions sont confirmées par les travaux du linguiste français Maurice Gross (1982, 151-152) qui a répertorié et classé plus de 60.000 phrases idiomatiques du français standard. Selon lui, les "phrases, formes ou expressions figées, encore qualifiées communément de proverbiales, idiomatiques ou composées » sont des phrases qui sont d'abord reconnues intuitivement et qui se caractérisent par le fait que «le sens des mots ne permet pas d’interpréter leur combinaison » (152).

Pour étudier le traitement que Perec a réservé aux phrases figées du français standard, nous ferons référence au cadre théorique du «lexique-grammaire » que Maurice Gross a développé en s'appuyant sur la théorie grammaticale transformationnelle développée par Zellig S. Harris (1976). Le point de départ de cette 
approche est la phrase simple et non pas le mot. À partir du lexique d'une langue donnée, on vérifie toutes les transformations possibles qu'une phrase peut subir en considérant les contraintes syntaxiques que la grammaire impose. Le but principal du « lexique-grammaire » est de décrire systématiquement une langue. À titre d'exemple, examinons, à l'aide de la terminologie du «lexique-grammaire », cette locution perecquienne réinventée qu'on lit dans $L a$ Disparition :

«Augustus B. Clifford a raccourci son chibouk» (Perec [1969] 1989, 136).

Cela permettra de voir quels sont les éléments qui peuvent éventuellement varier dans une expression figée. Derrière cette phrase perecquienne réinventée, on reconnait l'expression suivante attestée en français standard :

Augustus B. Clifford a cassé sa pipe.

Cette expression signifie que le personnage Augustus B. Clifford « est mort » (Ray-Chantreau 1989). Comme conséquence de la contrainte lipogrammatique, Perec ne pouvait pas utiliser l'expression cidessus, ni les expressions synonymes et/ou populaires comme " passer l'arme à gauche », " partir les pieds devant », " manger les pissenlits par la racine » ou « partir chez Saint-Pierre » car la voyelle « $E »$ apparaît plusieurs fois dans toutes ces structures. Il s'agit d'une expression sémantiquement opaque, son sens ne se trouvant ni dans le verbe "casser/raccourcir», ni dans le complément "pipe/chibouque ». D'après le Dictionnaire des expressions et locutions d'Alain Rey et Sophie Chantreau (1989), cette locution familière apparaît avec ce sens au milieu du XIX ${ }^{\mathrm{e}}$ siècle, mais on la retrouve également au XVII ${ }^{\text {e }}$ siècle dans les Mazarinades, avec le sens de « enrager».

Si l'on veut garder le sens figé de la locution «casser sa pipe $»^{2}$, on ne peut pas remplacer le verbe « casser » par d'autres verbes, ni le complément «pipe » par des synonymes comme Perec l'a fait :

Augustus B. Clifford a *(rompu + brisé + raccourci + détruit $)$ sa *(bouffarde + chibouque + son brûle-gueule).

Il n'est pas possible non plus de modifier les déterminants ou de modifier le complément avec un adjectif :

2 Nous utilisons plusieurs exemples de transformations lexicales et syntaxiques de la phrase figée « casser sa pipe » proposés par Maurice Gross $(1982,155)$. 
Augustus B. Clifford a cassé *(une + la + cette $)+*$ (vieille + nouvelle + élégante) pipe.

Les transformations qu'on peut apporter à cette phrase figée concernent le temps du verbe. On pourrait, en effet, considérer comme acceptable la phrase :

Augustus B. Clifford (cassa + cassera + aurait cassé) sa pipe.

Il est également possible d'insérer dans la phrase de départ, sans changer son caractère figé, des adverbes comme :

Augustus B. Clifford (cassa + a cassé + aurait cassé) sa pipe (il y a trois ans + soudainement).

Pour ce qui est de la syntaxe, on ne peut pas transformer la phrase de départ à la forme passive en disant :

* La pipe (est cassée + a été cassée) par Augustus B. Clifford.

De plus, si l'on veut maintenir le sens idiomatique, il est interdit de pronominaliser la phrase :

*Augustus B. Clifford a cassé sa pipe et Douglas Haig Clifford cassera la sienne.

Et, on ne peut non plus la rendre sous forme de proposition relative :

*La pipe qu’Augustus B. Clifford a cassée.

Les transformations d'habitude permises pour les phrases dites libres sont interdites dans les phrases figées. Toutefois, le roman étant en général un espace de liberté, dans La Disparition la contrainte devient pour Perec un moteur de création supplémentaire et surtout de subversion ludique de la langue. Le défigement des phrases figées opéré par l'écrivain illustre, selon nous, la volonté de vouloir "défiger » la réalité à l'aide d'un jeu habile de substitution des mots. Détourner les phrases figées, c'est réinventer la réalité de l'auteur que la disparition «d'E »3, «d'eux», (de ses parents) disparus comme le « $\mathrm{E} »$ dans le roman, a rendue tragique. Or, comment le traducteur italien Piero Falchetta a-t-il rendu dans la langue d'arrivée toutes les phrases détournées par l'écrivain ? Les a-t-il réinventées à l'image de l'auteur français ou

3 Voir la dédicace insérée par Perec dans $W$ ou le souvenir d'enfance. 
bien les a-t-il traduites en respectant simplement la contrainte du lipogramme perecquien?

\subsection{Stratégies de traduction en italien des phrases (dé)figées par Perec}

Analysons quelques exemples de près en partant de la phrase utilisée jusqu'ici comme modèle "Augustus B. Clifford a raccourci son chibouk », réinventée par l'écrivain oulipien ([1969] 1989, 136) à partir de la phrase figée " casser sa pipe » et cela pour respecter la contrainte lipogrammatique qu'il s'est imposée dans La Disparition. Pour cette phrase figée réinventée, le traducteur italien ne fait pas preuve de la même virtuosité linguistique que l'auteur. Bien qu'il respecte la contrainte, il la traduit par une phrase idiomatique attestée en italien, mais sans aucune réinvention :

"Il y a qu'Augustus B. Clifford a raccourci son chibouk il y a un instant » (Perec [1969] 1989, 136).

«Poco fa, Augustus B. Clifford ha giocato la sua ultima partita » (Perec/Falchetta 1995, 114).

Cette phrase idiomatique italienne empruntée au langage sportif, « jouer son dernier match », bien qu'elle véhicule le sens de mourir, « il trapasso da questa all'altra vita: la morte " (Vocabolario Treccani) (passer de vie à trépas : la mort), ne présente aucun défi d'interprétation pour les lecteurs italiens. Il s'agit d'une phrase idiomatique attestée qu'on utilise comme euphémisme à la place du verbe «mourir ». Aucune réécriture donc dans la langue d'arrivée, aucune acrobatie linguistique à l'image de Perec et surtout la perte de l'effet ludique que la phrase perecquienne provoque chez les lecteurs français.

La même stratégie visant à rendre moins lourd le texte dans la langue d'arrivée a été adoptée pour la traduction d'une autre phrase défigée. Dans le passage suivant, Perec propose encore une allusion amusante aux lecteurs français avec la phrase suivante défigée :

"Chacun gagna son local privatif, puis rapparu, un instant plus tard, mis sur son vingt-huit plus trois » (Perec [1969] 1989, 130).

"Ciascuno salì alla propria stanza, poi, dopo un po' tutti ritornarono abbigliati in pompa magna »(Perec/Falchetta 1995, 110).

Il apparaît que le lecteur italien ne pourra pas remarquer le travail sur la phrase opéré par l'auteur, car Piero Falchetta la traduit par l'équivalent italien attestée «vestirsi in pompa magna », 
" s'habiller en grande pompe », c'est-à-dire «solennellement » $(T L F i)$ et il ne réactive pas le même jeu que l'écrivain français a fait sur la phrase attestée en français. On remarque toutefois le choix, de la part du traducteur italien, du verbe pronominal « abbigliarsi » qui signifie, selon le Dizionario De Mauro, "vestirsi con cura ed eleganza », c'est-à-dire "s'habiller élégamment ». L'expression italienne choisie est redondante, mais elle appauvrit un petit peu la stratégie perecquienne, car il y a entropie au niveau métatextuel. Ce que l'écrivain oulipien appelait le «second niveau » de son roman n'est pas restitué dans la version italienne. Perec (1979, 48-49) luimême soulignait ceci à propos de cet aspect : " Dans La Disparition, il y a un personnage dont on ne pouvait écrire qu'il était mis sur son trente-et-un, alors j'ai écrit : "Mis sur son $28+3$ ”. Mais ça se situe à un second niveau. [...] Ce qui est important pour moi dans un livre, c'est qu'il puisse être lu à plusieurs niveaux ».

Pour traduire la phrase figée revisitée, le traducteur italien suit la même stratégie :

«Faisant ni six moins cinq, ni cinq moins trois, Quasimodo, d'un bond, sauta au fond du lac : l'hydrocution survint aussitôt » (Perec [1969] 1989, 252).

«In quattro quattr'otto Quasimodo si tuffò in fondo allo stagno: l'idrocottura fu cosa di un attimo » (Perec/Falchetta 1995, 205).

Le lecteur français, comme pour l'expression précédente, reconnaît sans problème l'expression idiomatique française attestée « ne faire ni une ni deux » qui signifie " ne pas hésiter, agir sur le champ » (TLFi). Cette réinvention perecquienne est très intéressante parce qu'elle illustre métatextuellement la conséquence de la contrainte lipogrammatique: le «moins 5 » de l'expression perecquienne évoque la disparition de la cinquième lettre de l'alphabet latin, c'est-à-dire le « $E$ ». En outre, 5 moins 3 font 2, c'està-dire la disparition de la deuxième voyelle, la lettre « $E$ ». De plus, le chiffre 3 renvoie dans La Disparition également à la voyelle disparue, car pour Perec il ressemble à la lettre « $E$ » reflétée dans un miroir.

Or, Piero Falchetta efface complètement cette illustration lipogrammatique contenue dans l'expression détournée par Perec car il utilise l'expression attestée en italien " in quattro e quattr'otto » qui signifie "in brevissimo spazio di tempo: in un attimo » (Vocabolario Treccani) et qui correspond à l'expression française « en moins de deux », c'est-à-dire "très rapidement » (TLFi). Il ne pouvait pas utiliser les chiffres italiens «sei » (6), « cinque » (5) et « tre » (3) car ils contiennent des « $E$ ». Il utilise alors une expression qui contient le chiffre « quattro » (4) sans tenir compte du chiffre 
« cinq » qui apparaît deux fois dans l'expression perecquienne. Les lecteurs de Perec savent toutefois que le chiffre « cinq » est thématisé tout au long du roman. Fidèle à la contrainte lipogrammatique, le traducteur italien élimine la lettre « $E$ » qui d'habitude lie les deux parties de l'expression « in quattro $e$ quattr'otto ».

Une autre expression métatextuellement connotée de l'auteur mérite notre attention, car le mot « blanc » y apparaît à la place du mot « brûle » :

«- Dis-moi, lui dit-il à blanc-pourpoint, pourquoi souris-tu? » (Perec [1969] 1989, 91).

«- Dimmi gli domandò di punto in bianco, cosa significa il tuo sorriso? » (Perec/Falchetta 1995, 76).

Tout lecteur français reconnaît sans difficulté l'expression attestée en français « dire à brûle-pourpoint » qui signifie « dire de très près, à bout portant » $(T L F i)$. Dans le roman de Perec, «le blanc " évoque le vide, la lacune, ce qui manque, mais aussi la disparition, bref la mort. Le blanc est également Moby Dick, le cygne (ou le signe), le Swann qui apparait souvent dans le roman et cela crée des renvois intertextuels à des chefs-d'œuvre de la littérature mondiale. Il s'agit d'un mot thématique et Piero Falchetta l'a bien rendu, puisqu'il a traduit l'expression détournée par Perec par l'expression idiomatique italienne « di punto in bianco » qui renvoie à « un atto compiuto o da compiere senza alcuna preparazione : così di punto in bianco non saprei decidere; abbandonò tutto di punto in bianco » (Vocabolario Treccani). L'expression française correspondante est " de but en blanc », qui signifie à "l'improviste, brusquement» (TLFi). On remarque que le sens des deux expressions n'est pas tout à fait pareil, mais le traducteur italien a choisi une expression contenant le mot «blanc» pour garder la cohérence métatextuelle. Comme le souligne Umberto Eco, la traduction s'inscrit sous le signe de la négociation permanente et dans l'exemple qu'on vient de citer, on voit bien comment le traducteur a dû négocier une légère perte de sens pour respecter la cohérence métatextuelle. En effet, la traduction d'un texte semblable sinscrit sous le signe du compromis permanent.

À ce point de notre analyse, nous pouvons confirmer l'hypothèse avancée plus haut : Perec a intentionnellement choisi des phrases figées avec la lettre « $E$ » pour les réinventer à travers son génie. La locution proverbiale suivante provoque également un sourire complice chez le lecteur qui doit ainsi coopérer à la construction d'un effet ludique tout en lisant un texte qui n'est pas du tout humoristique : 
«À malin, malin un quart» (Perec [1969] 1989, 235).

«Ora ti mostro io chi la sa più lunga » (Perec/Falchetta 1995, 193).

En effet, Perec réinvente la locution attestée « À malin, malin et demi » en remplaçant «demi » par «quart». Il s'agit d'un proverbe souvent cité de nos jours pour signifier qu'il existe «toujours plus malin que soi » (Rey-Chantreau, 1989). Toutefois, dans sa traduction italienne, Piero Falchetta normalise l'expression renouvelée par Perec en proposant la locution attestée "saperla lunga » qu'on utilise pour désigner quelqu'un qui est très rusé et qui, contrairement à ce qu'il veut faire croire, en sait beaucoup plus par rapport à ce qu'il dit : " furbo, o anche [che si sa] più di quanto si ha intenzione di dire » (Vocabolario Treccani). Aucun défigement dans le texte d'arrivée et par conséquent aucun sourire en coin chez les lecteurs italiens.

La locution proverbiale très ancienne, attestée chez Rabelais, " Oignez vilain, il vous poindra ; poignez vilain, il vous oindra », qu'on cite encore aujourd'hui pour signifier que « si vous traitez bien un individu grossier il vous manquera d'égards ; si vous le traitez mal, durement, il vous respectera » (Rey-Chantreau 1989) a également été détournée par Perec. Ce proverbe est réinventé grâce à un habile procédé de modification des verbes pivots « oignez/oignons » (enduire d'huile, mais métaphoriquement être gentil, doux) et " poignez/poignons » (blesser, maltraiter). De plus, Perec permute la place des segments en proposant un chassé-croisé de la locution attestée :

«Poignons vilain, il vous oindra,

Oignons vilain, il vous poindra!» (Perec [1969] 1989, p. 230).

« Non fidarti di un villano zoticaccio,

Tu gli dai la mano lui vorrà il tuo braccio! » (Perec/Falchetta 1995, 189).

Pour la traduction italienne de cette locution proverbiale ancienne, le traducteur propose une paraphrase en guise d'introduction au proverbe italien attesté " dare (un dito) una mano e farsi prendere (tutto) il braccio » qui signifie « abusare della libertà o della confidenza concessa » (Vocabolario Treccani). Ce proverbe italien est l'équivalent du proverbe français « Si on lui en donne long comme le doigt, il en prend long comme le bras ». On cite très souvent cette locution pour signifier que le destinataire du proverbe « abuse de la liberté [ou qu']il étend la permission qu'on lui accorde » (Dictionnaire de l'Académie, 1932-1935). 
Bien que Piero Falchetta respecte le lipogramme perecquien, le sens du proverbe qu'il propose comme traduction n'est pas le même que celui de l'expression détournée par Perec. Il existe en effet le proverbe italien très ancien « Il villano punge chi l'unge e unge chi lo punge » (Giusti, 1856) qui est l'équivalent du proverbe réinventé. À notre avis, cette locution attestée aurait pu être détournée par le traducteur à la manière de Perec et cela pour réactiver l'effet ludique que la manipulation habile de l'écrivain véhicule: par exemple «Pungi il villano, ti ungi, ungi il villano, ti pungi ». Une telle manipulation aurait créé une redondance qui aurait peut-être fait sourire les lecteurs italiens. Les expressions et les proverbes défigés gardent toujours la mémoire de la forme et du sens des expressions et des proverbes attestés, ce qui permet aux lecteurs de les reconnaître et d'en apprécier l'originalité du travail fait sur le lexique et sur la structure.

Nous insistons beaucoup sur l'aspect ludique du procédé mis en œuvre par l'auteur avec le détournement des phrases figées, car au-delà du sentiment tragique qu'on ressent à la lecture de chaque page du roman, La Disparition déploie également, grâce aux nombreux jeux de mots, une «spécificité du rire ». Julien Longhi $(2013,251)$ souligne à juste titre que

«le (sou)rire provoqué par les œuvres de Perec ne naît pas d'un contenu burlesque ou d'échanges comiques. Les thématiques abordées concernent des sujets délicats (disparition, totalitarisme), mais les contraintes formelles, qui aboutissent à un principe de lecture d'une forme ou figure sur un fond ludique, permettent le surgissement d'une jubilation interprétative. »

Or, nous croyons que cette "jubilation interprétative » ne surgit pas à la lecture des phrases figées dans La Scomparsa, puisque Piero Falchetta normalise les phrases détournées par Perec avant de les traduire en italien sans jouer le même jeu que l'écrivain oulipien.

Pour la traduction d'un autre proverbe réinventé par Perec, Piero Falchetta propose une traduction littérale en effaçant le caractère proverbial de l'expression...

«Tu troquas ton grimpant citadin pour un campagnard falzar, puis tu fis trois ablutions » (Perec [1969] 1989, 137).

"Cambiavi i tuoi pantaloni da città con un paio da campagna, poi ti bagnavi con 3 abluzioni » (Perec/Falchetta 1995, 115).

Perec détourne en effet le célèbre proverbe «troquer son cheval borgne pour un aveugle » qui signifie «faire une mauvaise affaire, perdre dans un troc » (Rey-Chantreau 1989). Piero Falchetta 
traduit mot à mot ce proverbe modifié et l'expression qu'il propose n'est pas attestée en italien. On connaît le proverbe italien équivalent "Cambiar un cavallo guercio con un cieco » ayant le même caractère sentencieux que le proverbe français. Ce proverbe italien véhicule, comme son équivalent français, le sens qu'on a conclu un mauvais marché. Cependant, dans la traduction proposée par le traducteur italien, on ne voit pas de réinvention par rapport à la phrase de départ et par conséquent il ne réactive pas le même jeu ludique que Perec avait mis en œuvre.

En réalité, il existe très peu d'exemples où le traducteur italien ait joué le même jeu que l'auteur français pour rendre une phrase figée réinventée. Dans la phrase qui suit, l'écrivain réécrit de la manière suivante l'expression idiomatique " prendre ses cliques et se claques » qui signifie «s'en aller brusquement en emportant son bien » $(T L F i)$ :

« Puis, instruit du sort qu'à coup sûr on m'allait garantir s'il m'attardait à partir, ayant pris mon clic sans avoir pour autant omis mon clac, j'ai fui Ankara, la maudissant à jamais » (Perec [1969] 1989, 274).

«Poi, istruitomi sull'infausto fato garantitomi qualora mi fossi attardato a partirmi, fatto prima quattro, ma non scordando di far quattrotto, scappai via da Ankara, apostrofandola di ingiuriosi insulti » (Perec/Falchetta 1995, 222).

De son côté, Piero Falchetta réécrit lui aussi l'expression idiomatique italienne " in quattro e quattrotto », c'est-à-dire " in brevissimo spazio di tempo : in un attimo » (Vocabolario Treccani), " en moins de deux », bref " très rapidement » (TLFi), en proposant un clin d'œil aux lecteurs italiens qui se rendent bien compte du jeu linguistique, puisque l'attente créée par la phrase figée n'est pas respectée et le côté ludique est réactivé en italien.

\section{Conclusion}

Bien que Piero Falchetta ait respecté la contrainte oulipienne, les exemples analysés montrent que le traducteur italien n'a pas joué tout à fait le même jeu que Perec dans la réinvention joviale des expressions figées. Comme Umberto Eco $(1983,17)$ l'a bien expliqué dans la préface de sa traduction des Exercices de style de Queneau : "Il s'agissait de décider ce que signifiait, à l'égard d'un livre de ce genre, être fidèle. Une chose était claire, c'est que cela ne voulait pas dire être littéral. [...] Être fidèle, cela signifiait comprendre les règles 
du jeu, les respecter, et puis jouer une nouvelle partie avec le même nombre de coups ${ }^{4}$ (traduction Bloomfield 2012, 132).

En réalité, la tâche n'était pas simple, puisque Piero Falchetta aurait dû opérer en tenant compte d'une contrainte supplémentaire. Pour la traduction des phrases défigées par Perec, en plus de la contrainte lipogrammatique, il aurait dû surmonter l'obstacle du figement pour proposer le même défi ludique aux lecteurs italiens.

Toutefois, le travail fait par l'écrivain sur le lexique des locutions figées fait surgir à plusieurs reprises un « amusement » qui se situe dans ce que Perec lui-même appelait le «second niveau » de son roman. Après l'analyse des traductions des phrases défigées, nous avons l'impression que ce "second niveau » de lecture prônée par Perec échappe considérablement aux lecteurs italiens. Nous rejoignons encore une fois, en guise de conclusion, la position de Julien Longhi $(2013,237)$ pour qui « le jeu des substitutions entre signifiants (ceux qui seraient utilisés sans la contrainte et ceux qui vont être finalement produits) constitue un fond ludique, fonde un contrat interprétatif pour la lecture, et les indices distillés par l'auteur rendent cette complicité tout autant nécessaire qu'appréciée, et provoquent le sourire, voire le rire, c'est-à-dire le (sou)rire perecquien ».

\section{Références bibliographiques}

BLOOMFIELD, Camille «Perec in Italia: inchiesta su una specie in via di moltiplicazione (Prima parte: Italia)», enquête auprès des traducteurs italiens de Perec, Georges Perec trent'anni dopo, I quaderni dell'Oplepo, $\mathrm{n}^{\circ}$ 2, Edizioni Oplepo, Naples, 2014, p.33-47. URL : www.academia.edu/10809955/

I_traduttori_di_Perec_inchiesta_su_una_specie_in_via_di_moltiplicazio ne_Prima_parte_Italia_(consulté le 17 janvier 2017).

BLOOMFIELD, Camille. « Traduire La Disparition de Georges Perec. Table ronde avec Valéri Kislov, John Lee, Vanda Mikšić, Marc Parayre et Shuichiro Shiotsuka ». In : Hélène Henry and Laurence Kiefé (dir.). Vingthuitièmes assises de la traduction littéraire. Arles : Coéditions Actes Sud, $2012: 129-156$.

ECO, Umberto. "Introduzione ». In : Raymond Queneau. Esercizi di stile. [Trad. italienne d'Umberto Eco]. Torino : Einaudi, 1983 : V-XIX.

GENETTE, Gérard. Palimpsestes. Paris : Seuil, 1982.

4 « Si trattava, in conclusione di decidere cosa significasse, per un libro del genere, essere fedeli. Ciò che era chiaro è che non voleva dire essere letterali [...]. Fedeltà significava capire le regole del gioco, rispettarle, e poi giocare una nuova partita con lo stesso numero di mosse ». 
GREAVES, Sara. «Une traduction non plausible!». Palimpsestes. «Traduire la littérature des Caraïbes. La plausibilité d'une traduction : le cas de La Disparition de Perec », 12, 2000 : 103-116.

GROSS, Maurice. "Une classification des phrases figées du français ». Revue Québécoise de Linguistique, vol. 11, $\mathrm{n}^{\circ}$ 2, 1982 : 151-185.

HARRIS, Zellig Sabbettai. Notes du cours de syntaxe. Paris : Seuil, 1976.

LONGHI, Julien. "Modalités et portées du (sou)rire chez Georges Perec ». In : Violaine Houdart-Merot (dir.). Rires en francophonie. Amiens, Encrage édition, coll. « CRTF », $2013:$ 235-252.

MATHEWS, Harry. "Reviews La Disparition by Georges Perec ». American Book Review, 3 : 6 octobre 1981: 29-35.

PEREC, Georges. "En dialogue avec l'époque ». Entretien avec Patrice Fardeau. France Nouvelle, n $^{\circ} 1744$ (16-22 avril 1979). In : Dominique Bertelli et Mireille Ribière (Édition établie par).Georges Perec en dialogue avec l'époque et autres entretiens. Nantes : Joseph K., 2011.

ROUBAUD, Jacques. Atlas de littérature potentielle. Paris: Gallimard, 1988.

\section{Dictionnaires}

Dizionario De Mauro. Torino: Paravia, 2001. URL : http://dizionario.internazionale.it. (Consulté le 3 janvier 2017).

Dictionnaire de L'Académie française (8e édition). Paris: Imprimerie Nationale/Fayard, 1935. URL : http://atilf.atilf.fr/academie.htm. (Consulté le 17 janvier 2017).

DUBOIS, Jacques et alii. Dictionnaire de linguistique et des sciences du langage. Paris : Larousse, 1994.

GIUSTI, Giuseppe. Dizionario dei proverbi italiani. Milano : L. Veronelli, 1856.

Le Nouveau Petit Robert. Rey-Debove Josette et Rey Alain (dir.), Paris : Dictionnaires Le Robert, 1996.

REY, Alain, Sophie Chantreau. Dictionnaire des expressions et locutions. Paris : Les Usuels du Robert, coll. «Poche », 1989.

Trésor de la Langue Française informatisé. Paris : CNRS/Gallimard, 1994. URL : http://atilf.atilf.fr/. (Consulté le 3 janvier 2017).

Vocabolario Treccani e Dizionario di Italiano Garzanti Linguistica. Roma, Istituto dell'Enciclopedia Italiana. 2010. Disponibile on line: URL: www.treccani.it/vocabolario.

VOLPINI, Carlo. 516 Proverbi sul cavallo. [516 Proverbes sur le cheval]. Milano : Cisalpino Goliardica, 1984.

\section{Textes de référence}

PEREC, Georges

W ou le souvenir d'enfance. Paris : Denoël, 1975.

La Disparition. Paris : Gallimard, coll. "L’Imaginaire », 1989 [1969].

La Scomparsa. [Trad. italienne de Piero Falchetta]. Napoli : Liguori, 1995. 